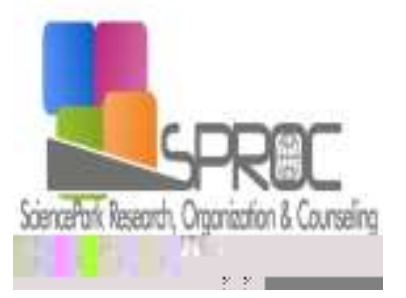

\section{New Trends and Issues Proceedings on Humanities and Social Sciences}

Volume 4, Issue 1 (2017) 323-328
'New Trends and Issues Proceedings on Humanities and Social Sciences

ISSN 2421-8030

www.prosoc.eu

Selected Papers of 9th World Conference on Educational Sciences (WCES-2017) 01-04 February 2017 Hotel Aston La Scala

Convention Center, Nice, France

\title{
An interactive " $3 S$ " obesity education program among married women in Kelantan, Malaysia: Does it effective?
}

\footnotetext{
Nazihah Mohd Yunus ${ }^{a^{*}}$, School of Medical Sciences, Health Campus Kota University Sains, Bahru, 16150, Kelantan, Malaysia.

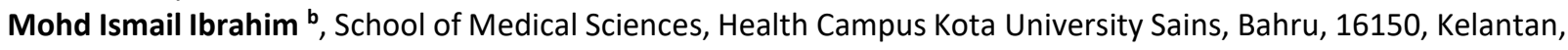
Malaysia.
}

\section{Suggested Citation:}

Yunus, M. N. \& Ibrahim, I. M. (2017). An interactive "3S" obesity education program among married women in Kelantan, Malaysia: does it effective?.

[Online]. 4(1), pp 323-328. Available from: www.prosoc.eu

Selection and peer review under responsibility of Prof. Dr. Jesus Garcia Laborda, University of Alcala, Spain. ${ }^{\circ} 2017$ SciencePark Research, Organization \& Counseling. All rights reserved.

\begin{abstract}
The aim of the study was to determine the effectiveness of the "3S" (Simple, Sustainable and Suitability) Obesity Education Program by assessing the KAP scores. It was a community intervention study involving obese and overweight married women in suburban area of Kelantan, Malaysia. BMI level, and KAP scores, level of perception of body image and the need assessment on interactive 3S Education program were determined as baseline measurement. Post assessment was done 4 weeks later. A hundred participants were involved and majority of them had poor perception on body image, but willing to participate. The difference in mean scores of each KAP domain were statistically significant respectively [Knowledge score: $24.21,26.80, p=$ 0.005, Attitude score: 5.70, 8.30, $p<0.001$, Practice score: $6.62,9.32, p=0.002$ ] and the increment of mean score more than

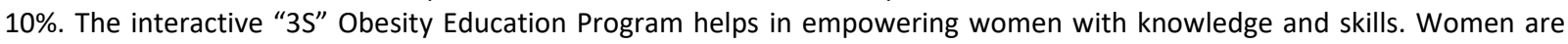
recommended to use it since they play major role in providing healthy diet to their family members.
\end{abstract}

Keywords: Obesity; interactive education program; married women; suburban area.

\footnotetext{
* ADDRESS FOR CORRESPONDENCE: Nazihah Mohd Yunus, School of Medical Sciences, Health Campus Kota University Sains, Bahru, 16150, Kelantan, Malaysia.
} 
Yunus, M. N. \& Ibrahim, I. M. (2017). An interactive "3S" obesity education program among married women in Kelantan, Malaysia: does it effective?.

[Online]. 4(1), pp 323-328.

Available from: www.prosoc.eu

\section{Introduction}

Obesity is epidemic worldwide. It affects all level of community including children at preschool. The condition is strongly associated with increasing trend of non-communicable diseases like diabetic mellitus at young age group, heart attack and stroke. Those diseases carry significant burden to the country and lead to reduce number of talented human resource due to premature death (Swinburn et al., 2011). Thus, prevention is better than cure. It is fortunate to understand that obesity is a preventable disease but for those who are already obese, it is not an easy task to get back to ordinary body shape or have ideal body weight (Ofei, 2005).

For society in Asian countries such as Malaysia, married women play an important role in the preparation and selection of food to her family members. Even though some of them are career women, they still have to prepare the food and find the best menu to serve every member of their family. The majority of the people in Kelantan are Malay. Their cuisine is somewhat influenced by Indian culture. They use coconut milk as the main ingredient in cooking (Noraziah \& Mohd-Azlan, 2012). Financial constraints and lack of knowledge and skills leading to many of them use non-nutritive substances in the preparation of the main menu (Drewnowski \& Spectra, 2004). This causes the prevalence of obesity among them increased significantly.

There are many health education models that can be used to help the community to understand the health problem and try to prevent the disease from occurring. The concept used usually related to the belief and perceives understanding of the subjects. Health belief model for example is used to evaluate the barrier and benefits of taking action (James et al., 2012). Understanding the variables such as norms and belief of the targeted group will help the researcher to identify factors that hinder and promote them to reduce the prevalence of the problem such as obesity (Caprio et al., 2008). Understanding their culture and nature of their daily food intake could give the researcher idea in conceptualizing the

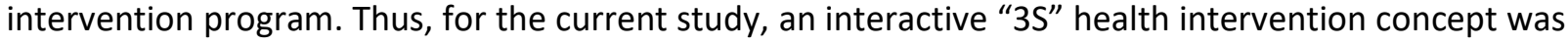
developed. The aim of the intervention is to ensure the content is sustainable, suitable and simple, which mean it needs no complicated instrument or ingredients. The objective of the current study is to look at the effectiveness of the intervention packages by assessing the knowledge, attitude and practice of the married women on the obesity preventive measure.

\section{Methods and Tool}

Kg. Sokor is located at Daerah Ketereh, Kelantan. It is $2.3 \mathrm{~km}^{2}$ areas. There are 149 houses consists of 689 residents. Majority of the villagers are Malay, Muslims and speak Kelantanese dialect. Kg Sokor is located $75 \mathrm{~km}$ from Kota Bharu, the capital city of Kelantan. Kelantan itself is one of the north-east states in Peninsular Malaysia.

This was a community intervention study. It was started from September 2013 for four months period. The intervention took place for a month and post intervention was carried out a month after intervention.

The study involved married women who permanently stay in the selected village (Kg Sokor). The exclusion criteria include those who are pregnant and not overweight or obese during study period. The targeted group was identified during the assessment on community profile and assessment on perception of self body image. Those who fulfill the study criteria were asked about their willingness to 
Yunus, M. N. \& Ibrahim, I. M. (2017). An interactive "3S" obesity education program among married women in Kelantan, Malaysia: does it effective?.

[Online]. 4(1), pp 323-328.

Available from: www.prosoc.eu

fully participate in the intervention program. If they could not commit to spare their time for the program, they were excluded from the study.

The sample size was calculated by using two mean formula whereby the minimum number of sample requires to achieve the objective of the study was 123 person. Since the number of available participant that fulfill the study criteria was smaller than the required sample, the study only could apply non purposive sampling method. All of them who fulfill the study criteria were included.

A set of validated Malay version questionnaire was used. It was divided into 2 parts: Part I was subdivided into two areas namely Part A and B. Part A consists of respondent socio-demographic characteristics such as age, sex, race, level of knowledge, occupation and income while Part B consists of the anthropometry measurement like weight, height, waist and hip ratio circumference. For the purpose of the current study, WHO BMI classification was used.

Part II of the questionnaire consists of 3 sub-division namely Part A, B and C. Part A was regarding the knowledge about obesity example the risk factor, complication and ways to prevent obesity, dietary knowledge and proper ways to reduce body weight. This part consists of 39 items and was scored as 1 mark for correct answer and 0 mark for incorrect or not sure answer. Part B contains 14 items basically related to attitude towards self-awareness and self-control of their body weight. The scoring system was 1 mark for good attitude and 0 mark for poor attitude. Part $C$ was developed to assess practice related to eating habits and cooking method which contains 11 items in total. The score was given as follow, 1 mark for good practice and 0 mark for bad practice.

It consists of activities as follow:

- Health talk - it was delivered by nutritionist and doctor in Kelantanese dialect. It is to ensure the participants understand more the content and encourage two ways interaction. The use of IT in the program, had enhanced the interaction between participant and speaker, the animation and illustration in the form of power point make them feel real and able to visualize the problem much better.

- $\quad$ Cooking demonstration - to make it more interesting, the demonstration was done in form of competition. In the beginning, the expert was invited to show how to cook a healthy diet using ingredients that often used by respondents such as bamboo shoots, banana, freshwater fish, etc. the way of cooking was modified. They were shown steps to improvise their cooking skill from fried to steam, from steam to grill etc. Once they understood the process, a cooking competition was organized.

- $\quad$ Aerobics exercise - to ensure the sustainability of the activity, a local influence person was appointed to lead the team. She was trained how to conduct the aerobic exercise by the appointed aerobics instructor. The exercise was conducted every Friday and Saturday morning.

- Home party - this concept was introduce to help those who unable to come or miss the event. A group of research assistant was identified to go to the houses that were indentified. Those who stay 
Yunus, M. N. \& Ibrahim, I. M. (2017). An interactive "3S" obesity education program among married women in Kelantan, Malaysia: does it effective?.

[Online]. 4(1), pp 323-328.

Available from: www.prosoc.eu

around the house were invited to join the "party". The activities include health talk and discussion on the issue raise.

- Providing the Recipe Book and Booklet regarding obesity - these two items are used to ensure the knowledge and information that been taught is sustained in the community.

Data were entered and analyzed using SPSS version 20. The data were checked and cleaned. Data analyses were done using numerical data (mean and standard deviation) and categorical data (frequency and percentage). The significant level value was 0.05 . Statistical test were done using numerical data analysis (Paired t test) which is used for the analysis of mean knowledge, attitude and practice scores obtained pre and post intervention.

\section{Results}

A total of hundred participants enrolled in the study. Their mean age, BMI score and house whole income per month were 49.1 (15.64), 29.6 (10.23), RM1,452 (945.34) respectively. Majority of them were housewife and had completed the secondary school level. Pre intervention assessment revealed that majority of them had poor perception on their own body image whereby $60 \%$ perceive that being obese does not affect their appearance in the community. However, in the same time, the finding also revealed that they have strong will to participate in the obesity intervention program.

In relation to the mean KAP score pre and post intervention, the study revealed that in all domains, their mean score different was statistically significant. The knowledge score improved from 24.21 to 26.80 and the attitude score climbed up from 5.70 to 8.30 while the practice score increased from 6.62 to 9.32 (Table 1).

Table 1. The KAP score pre and post intervention $(n=100)$

\begin{tabular}{cccccc}
\hline Variables & $\begin{array}{c}\text { Pre-Test } \\
{[\text { mean (SD)] }}\end{array}$ & $\begin{array}{c}\text { Post-Test } \\
\text { [mean (SD)] }\end{array}$ & $\begin{array}{c}\text { Mean difference } \\
(95 \% \text { CI) }\end{array}$ & \% inclination & P value* \\
\hline Knowledge score & $24.21(6.46)$ & $26.80(6.39)$ & 2.59 & 10.7 & 0.005 \\
Attitude score & $5.70(2.13)$ & $8.30(2.08)$ & $(0.82-4.35)$ & 2.60 & $<5.6$ \\
Practice score & $6.62(1.26)$ & $9.32(6.51)$ & $\begin{array}{c}(1.96-3.24) \\
2.70\end{array}$ & 40.8 & $<0.001$ \\
\hline
\end{tabular}

\section{Discussion}

The current study shows that the respondents gained adequate knowledge on general aspect of obesity, its risk factors and complications as well as steps in the preventive measure. The mean score improved as much as $10.7 \%$. This could be due to the multi strategy community projects which are simple but effective and practical can improve knowledge and skill of the community. As mention by a previous study, the most effective public health education programs are those that address or overcome issues specific to the intended target population. One substantial issue is culture. A recent study has taken into account issues relating to norms, belief and perception of the targeted group prior starting the program (Caprio et al., 2008). Despite that, the use of local dialect also play significant role in this project. For those who come from low education and socioeconomic background, they might feel easier to understand the content of talk and to follow the instruction if it was conducted in local dialect.

Despite an inclination of mean knowledge score, the result also shows an increment of attitude score of more than $10 \%$. The finding indicates a good sign that the intervention had changed their perception 
Yunus, M. N. \& Ibrahim, I. M. (2017). An interactive "3S" obesity education program among married women in Kelantan, Malaysia: does it effective?.

[Online]. 4(1), pp 323-328.

Available from: www.prosoc.eu

on obesity. Before joining the intervention program, most of them have misconceptions about their body image. They do not feel that obesity should be prevented and treated. After intervention, they knew that the obesity is a disease and it leads to many other diseases like diabetes, hypertension, cancer and stroke. Most importantly, they knew that obesity is a preventable disease. As a result of good attitude, majority of the respondents agreed that they should monitor their body weight from time to time. According to a study by Ronda et. al. (2001), respondents who were not aware of their inadequate physical activity level had a less positive intention to increase their level of physical activity. The interactive $3 S$ intervention program has proven to be able to inculcate positive attitude among the respondents.

The total mean score of practice regarding obesity increase from 6.62 during pre-intervention to 9.32 during post intervention. The percentage significantly increased by $40.8 \%$. It shows the effectiveness of the program in improving the practical skill of the respondents. The use of multiple approaches and the application of respondents centre focus had given positive impact to entire KAP scores. As reported by a previous study, the score of practice increases when the intervention program is multifaceted, specifically community-based, has high potential for sustainability, and hence effective (Middleton et al., 2014). The booklet we provided is user friendly, easily understand, acceptable and picture-guided. We received good feedback from respondents about the Exercise Diary handout that guide them explicitly and help them to be disciplined in performing physical exercise as mention in previous literature, an explicit guidance contributes to the increase in practice score (Catford, 2009). The establishment of the women aerobics club provides a platform for them to exercise healthy practices with a group of people with similar objectives. Jolly et al. (2011) found that groups such as this are very effective in weight management. Thus, they apply the healthy practices in their daily life such as regular exercise, and choose and prepare healthy food for their family.

\section{Conclusion and Recommendation}

An interactive " $3 S^{\prime}$ " obesity intervention program has shown to be effective in empowering the married women with knowledge, attitude and practical skill. It helps the targeted group to understand more about obesity and move forward to prevent the disease from occurring at least among their family members.

\section{Acknowledgement}

We would like to thanks all the participants and villages in Kg Sokor who directly or indirectly involved in the study. We also would like to extend our sincere thanks to the Universiti Sains Malaysia for providing fund to conduct the study via Community and Family Case Study (CFCS) Program. To all medical students from group G academic session 2013/2014, thank you and well done. 
Yunus, M. N. \& Ibrahim, I. M. (2017). An interactive "3S" obesity education program among married women in Kelantan, Malaysia: does it effective?.

[Online]. 4(1), pp 323-328.

Available from: www.prosoc.eu

\section{References}

Caprio, S., Daniels, S. R., Drewnowski, A., Kaufman, F. R., Palinkas, L. A., Rosenbloom, A. L. \& Kirkman, M. S. (2008). Influence of race, ethnicity, and culture on childhood obesity: Implications for prevention and treatment. (12), 2566-2577.

Catford, J. (2009). Advancing the "scince of delivery" of health promotion: Not just the "science of discovery". (1), 1-5.

Drewnowski, A. \& Specter, S. E. (2004). Poverty and obesity: the role of energy density and energy costs. The (1), 6-16.

James, D. C., Pobee, J. W., Brown, L. \& Joshi, G. (2012). Using the health belief model to develop culturally appropriate weight-management materials for African-American women.

(5), 664-670.

Jolly, K., Lewis, A., Beach, J., Denley, J., Adab, P., Deeks, J. J. \& Aveyard, P. (2012). Comparison of range of commercial or primary care led weight reduction programmes with minimal intervention control for weight loss in obesity: lighten up randomised controlled trial.

(3), 286287.

Middleton, G., Henderson, H. \& Evans, D. (2013). Implementing a community-based obesity prevention programme: experiences of stakeholders in the north east of England. 201-211.

Noraziah A. \& Mohd Azlan A. (2012). The food consumption and eating behaviour of Malaysian urbanites: Issues and concerns.

Ofei, F. (2005). Obesity-a preventable disease. , (6), 157-165.

Ronda, G., Van Assema, P. \& Brug, J. (2001). Stages of change, physchological factors and awarness of physical activity levels in the Neteherland.

, (4), 305-314

Swinburn, B. A., Sacks, G., Hall, K. D., McPherson, K., Finegood, D. T., Moodie, M. L. \& Gortmaker, S. L. (2011). The global obesity pandemic: shaped by global drivers and local environments.

(9793), 804-814. 